\title{
High-Frequency Subsurface and Bulk Dynamics of Liquid Indium
}

\author{
H. Reichert, ${ }^{1}$ F. Bencivenga, ${ }^{2}$ B. Wehinger, ${ }^{1}$ M. Krisch, ${ }^{2}$ F. Sette, ${ }^{2}$ and H. Dosch ${ }^{1}$ \\ ${ }^{1}$ Max Planck Institut für Metallforschung, Heisenbergstrasse 3, D-70569 Stuttgart, Germany \\ ${ }^{2}$ European Synchrotron Radiation Facility, Boîte Postale 220, F-38043 Grenoble Cedex, France
}

(Received 8 September 2006; published 28 February 2007)

\begin{abstract}
We have performed bulk and surface-sensitive inelastic x-ray scattering experiments on liquid indium with $3 \mathrm{meV}$ energy resolution. The experimental data are well reproduced within a generalized hydrodynamic model including structural and microscopic relaxation processes. We find a longitudinal viscosity of $22 \mathrm{mPa} s$ in the near-surface region compared to $7.4 \mathrm{mPa} \mathrm{s}$ in the bulk. The origin of the increase is associated with a slowing down of the collective dynamics in a subsurface region of $4.6 \mathrm{~nm}$.
\end{abstract}

DOI: 10.1103/PhysRevLett.98.096104

PACS numbers: 68.03. $-\mathrm{g}, 63.22 .+\mathrm{m}, 68.35 . \mathrm{Ja}$

Liquid metals reveal a host of intriguing surface phenomena, such as atomic layering, density fluctuations perpendicular to the surface (capillary waves), or peculiar ordering phenomena in alloys [1-3]. In particular, the static (time-averaged) aspects of the structure of liquid metals have been the subject of numerous studies and are well documented, both for the bulk and the surface [4]. On the other hand, the influence of these surface phenomena on the dynamics in the near-surface region is largely unknown, in particular, at short length scales.

The dynamics in bulk liquids at interatomic length scales can be studied by inelastic neutron (INS) and $\mathrm{x}$-ray scattering (IXS) techniques, and many important insights have been gained concerning the nature of acousticlike (propagating) excitations, their relation to the local atomic structure, and the influence of relaxation phenomena on the excitation spectrum [5,6]. In contrast to this, the high-frequency dynamics of liquid surfaces and interfaces has not been experimentally studied due to the lack of suitable experimental techniques.

In this Letter we present results on the bulk and subsurface dynamics of liquid indium obtained by IXS. An analysis within the framework of a generalized Langevin formalism reveals differences in the dispersion of longitudinal collective modes. Most significantly, we found an increase of the longitudinal viscosity in the subsurface region. Our findings indicate that this high surface viscosity arises from the slowing down of the dynamics related to the collective rearrangements of the local structure.

The experiment was carried out on the beam line IXS II (ID28) at the European Synchrotron Radiation Facility in Grenoble, France. The instrument was operated using mostly the $\mathrm{Si}(999)$ configuration, with an incident photon energy of $17.794 \mathrm{keV}$ and a total instrumental energy resolution of $3 \mathrm{meV}$ full-width-half-maximum (FWHM). For the bulk measurements a highly pure indium foil of $50 \mu \mathrm{m}$ thickness - corresponding to one absorption length - was loaded in a resistively heated cell with single crystal diamond windows, and placed into a vacuum chamber. A subset of data was recorded with a higher energy resolution of $1.7 \mathrm{meV}$ FWHM (incident photon energy $21.747 \mathrm{keV}$ ) in order to check the consistency of our analysis. For the IXS measurements in surface-sensitive geometry the $\mathrm{x}$-ray beam dimensions of $250 \times 60 \mu \mathrm{m}^{2}$ (horizontal $\times$ vertical, FWHM) were further reduced in the vertical direction by a slit to $40 \mu \mathrm{m}$ in order to limit the footprint on the sample to $23 \mathrm{~mm}$ such that the energy resolution is not affected by the footprint of the beam on the sample. The incident $\mathrm{x}$-ray beam with a divergence of $120 \times 60 \mathrm{mrad}^{2}$ (hor. $\times$ vert.) was deflected by a platinum coated mirror and impinged with a glancing angle of $0.098^{\circ}$ onto the sample.

The sample for the surface-sensitive measurements was highly pure indium kept in a high purity molybdenum trough with a diameter of $55 \mathrm{~mm}$ at a temperature of $170^{\circ} \mathrm{C}$. Because of the large sample diameter, the sample was sufficiently flat in the center for the grazing angle scattering geometry. The sample container was resistively heated and the temperature was monitored by a NiCr-Ni thermocouple. The sample assembly was mounted in a specially designed compact UHV chamber at a base pressure of $2 \times 10^{-10}$ mbar equipped with a cold cathode $\mathrm{Ar}$ ion sputter gun. The sample was cleaned by prolonged cycles of sputtering and heating over the course of several days and once during the experiment.

For the IXS measurements the UHV chamber was mounted on a vibration-isolation stage, which was fixed on the diffraction stage of the inelastic scattering setup. Throughout the experiments the incident angle $\alpha_{i}$ of the xray beam was kept below the critical angle of total external reflection, $\alpha_{c}=0.16^{\circ}$. Figure 1 shows a sketch of the setup and the scattering geometry with the momentum transfer $Q$ parallel to the surface. In this geometry, the scattered radiation was collected for an exit angle of $0.03 \alpha_{c} \leq \alpha_{f} \leq 3.4 \alpha_{c}$ with respect to the sample surface, corresponding to a probed sample depth of $\Lambda=4.6 \mathrm{~nm}$ at an incident angle of $\alpha_{i}=0.61 \alpha_{c}$, where the contribution to the scattered intensity has dropped to $1 / e$. For both setups the scattered beam was energy analyzed by a set of 5 analyzers at a backscattering angle of the analyzers of 


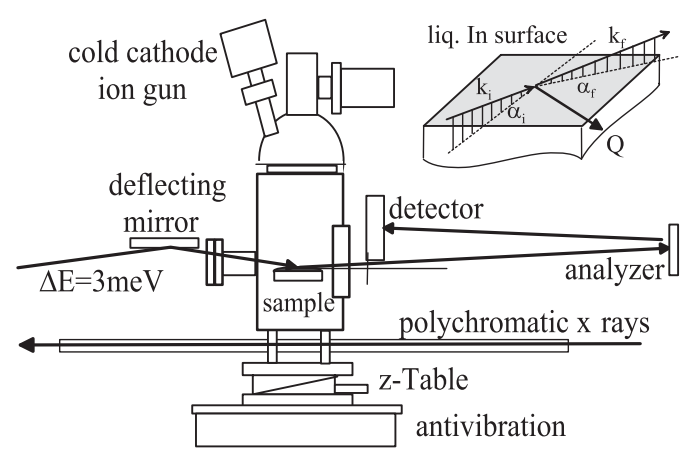

FIG. 1. Sketch of the experimental setup. The incoming polychromatic x-rays are backscattered with an energy resolution $\triangle E$ of $3 \mathrm{meV}$ towards the sample which is kept in an UHV chamber. The scattered radiation is detected after backscattering from a multianalyzer setup.

$89.983^{\circ}$ and an arm length of $6.5 \mathrm{~m}$, allowing for the simultaneous collection of five spectra with a $Q$ resolution of $0.24 \mathrm{~nm}^{-1}$.

In Fig. 2 we report representative IXS spectra in bulk (left panels) and surface-sensitive geometry (right panels). The spectra display the characteristics of the Brillouin triplet: a central line and two symmetric inelastic features related to longitudinal sound excitations. In both cases the dispersion with momentum transfer, $Q$, and the increasing energy spread of the sound mode is clearly visible.

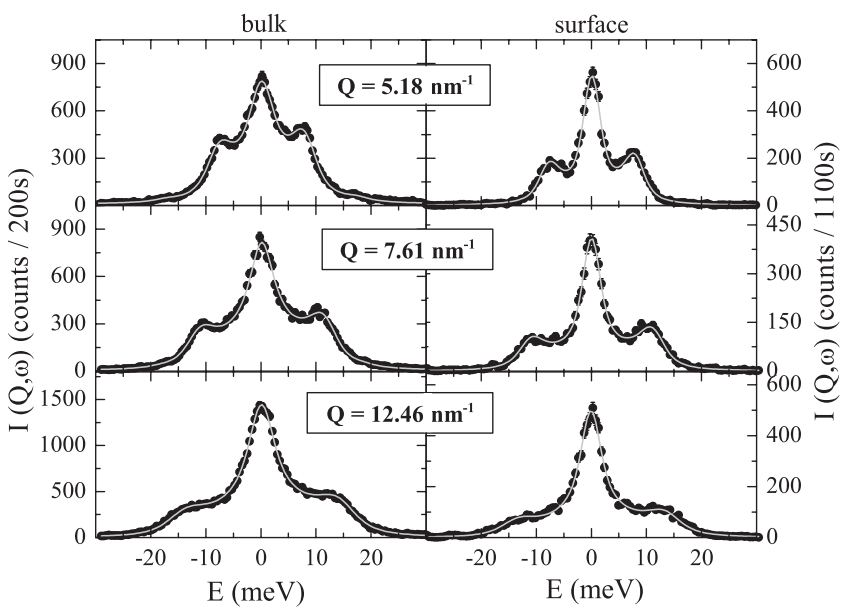

FIG. 2. Representative IXS spectra of liquid indium in bulk (left panels) and surface-sensitive geometry (right panels) at the indicated momentum transfer values $Q$. The experimental data (black circles) and their respective error bars are shown together with the best fit results using a model function as described in the text.

The data were analyzed within the framework of generalized hydrodynamics, using the memory function approach. In this frame the classical dynamic structure factor reads $[7,8]$

$$
\frac{S(Q, \omega)}{S(Q)}=\frac{1}{\pi} \frac{\Omega_{0}^{2}(Q) M^{\prime}(Q, \omega)}{\left[\omega^{2}-\Omega_{0}^{2}(Q)-\omega M^{\prime \prime}(Q, \omega)\right]^{2}+\left[\omega M^{\prime}(Q, \omega)\right]^{2}} .
$$

Here $M^{\prime}(Q, \omega)$ and $M^{\prime \prime}(Q, \omega)$ are, respectively, the real and imaginary part of the Fourier transform of the memory function: $M(Q, t) ; k_{B}$ is Boltzmann's constant, $S(Q)$ the static structure factor, and $m$ the indium atomic mass. The quantity $\Omega_{0}^{2}(Q)=k_{B} T Q^{2} /[m S(Q)]$ is related to the generalized isothermal sound velocity through the relation $c_{T}(Q)=\Omega_{0}(Q) / Q$. The $S(Q, \omega)$ in Eq. (1) was modified to satisfy the detailed balance condition and convoluted with the instrumental resolution function, $R(\omega)$, such that the relevant parameters can be directly extracted from a fit to the experimental spectra:

$$
I(Q, \omega)=A x[n(x)+1] S(Q, \omega) \otimes R(\omega),
$$

where $\otimes$ is the convolution operator, $A$ is a scaling factor, $n(x)$ is the Bose factor, and $x=\hbar \omega / k_{B} T$.

In analogy to previous IXS studies on liquid metals [912 ], we chose a memory function composed of three exponential decays, each one accounting for a different relaxation process:

$$
\begin{aligned}
M(Q, t)= & \Delta_{T}^{2}(Q) e^{-t / \tau_{T}(Q)}+\Delta_{\alpha}^{2}(Q) e^{-t / \tau_{\alpha}(Q)} \\
& +\Delta_{\mu}^{2}(Q) e^{-t / \tau_{\mu}(Q)} .
\end{aligned}
$$

$\Delta_{T, \alpha, \mu}^{2}$ are the strengths of the thermal, structural, and microscopic relaxation, respectively, while $\tau_{T, \alpha, \mu}$ are the corresponding relaxation times. The microscopic relaxation takes into account the short time decay of the memory function. In simple monatomic systems it is associated to the topological disorder that, in the investigated $Q$ range, enables the identification of the system's eigenstates with plane waves [13]. As a consequence, an experimentally excited plane wave is projected into different eigenstates with different eigenvalues (frequencies). These eigenstates, after a certain characteristic time, $\tau_{\mu}$, dephase with each other. This mechanism leads to a relaxation process, since it can be regarded as an energy transfer, taking place over a characteristic time scale, of the excited wave in favor of other eigenstates of the system. The structural relaxation instead accounts for the energy exchanges associated with collective rearrangements of the local structure by means of intermolecular interactions (i.e., bonding or "correlated collisions"). Finally, the thermal relaxation rules the energy exchanges related to heat flow. In the case of liquid indium, this relaxation process can be neglected because: (i) Its dispersive effect takes place at $Q^{*} \cong c_{s} / D_{T}$, where $c_{s}$ and $D_{T}$ are the adiabatic sound velocity and the thermal diffusion coefficient [6], respectively. With $c_{s} \sim 2300 \mathrm{~m} / \mathrm{s}$ and $D_{T} \sim 23 \mathrm{~nm}^{2} / \mathrm{ps}$ [14,15] for liquid indium, we find $Q^{*} \sim 0.1 \mathrm{~nm}^{-1}$, a $Q$ value definitely lower than the probed ones. (ii) In the fully 
relaxed limit of the thermal relaxation, its contribution to the mode damping $\left(\Gamma_{T}=\Delta_{T}^{2}(Q) \tau_{T}(Q) \sim 0.02 \mathrm{meV}\right)$ is much smaller than the experimental resolution function.

The model function therefore contains 6 fit parameters: $\tau_{\alpha}(Q), \tau_{\mu}(Q), \Delta_{\alpha}^{2}(Q), \Delta_{\mu}^{2}(Q), A$ and the low-frequency (isothermal) dispersion, $\Omega_{0}(Q)$. This function describes the experimental spectra very well as Fig. 2 demonstrates. This is reflected in the $\chi^{2}$ values of about one for all the fit results. Once the fits have been performed, one can also calculate the infinite-frequency limit of the dispersion relation, $\Omega_{\infty}(Q)$, via the relation: $\Omega_{\infty}(Q)=$ $\sqrt{\Omega_{0}^{2}(Q)+\Delta_{\alpha}^{2}(Q)+\Delta_{\mu}^{2}(Q)}$.

The other fundamental information one can derive from the IXS spectra is the characteristic frequency of the longitudinal sound mode: $\Omega_{L}(Q)$. This represents the maxima of the longitudinal current spectrum, $J_{L}(Q, \omega)=$ $\omega^{2} S(Q, \omega)$. For lower $Q$ values, namely, $2.75 \mathrm{~nm}^{-1}$ and $4 \mathrm{~nm}^{-1}$, the correlation among the fitting parameters is too high to produce a reliable fit. In these cases the line shape model function of Eqs. (1)-(3) has been replaced by a Lorentzian function centered at $\omega=0$ plus a damped harmonic oscillator function, whose maxima are considered to be equal to the maxima of $J_{L}(Q, \omega)$.

Figure 3 reports the isothermal (circles), longitudinal (diamonds), and infinite-frequency (triangles) sound dispersion in bulk (open symbols) and surface (full symbols) geometry. We note an excellent agreement for the bulk data set recorded with two different energy resolutions. We also note that the longitudinal dispersion is systematically

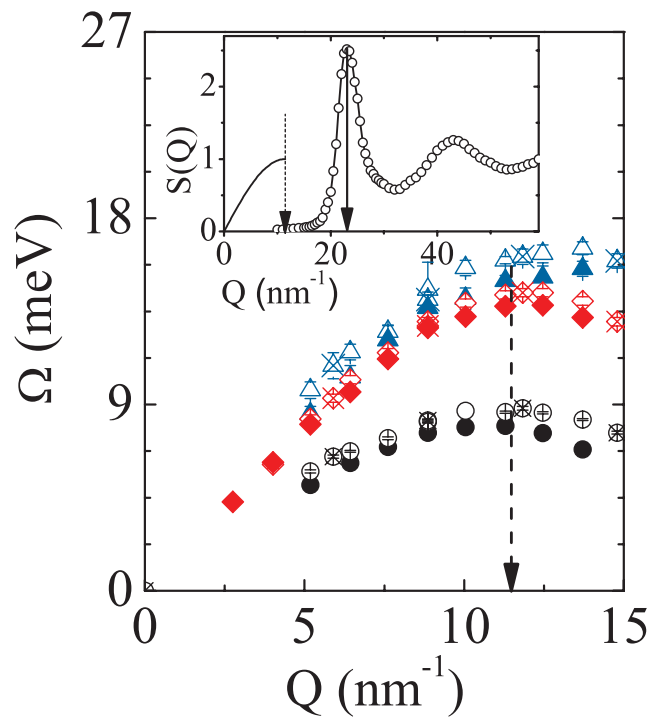

FIG. 3 (color). Longitudinal (red diamonds), isothermal (black circles), and infinite-frequency (blue triangles) sound dispersions of liquid indium in bulk (open symbols) and surface-sensitive geometry (full symbols). The bulk results obtained with a higher energy resolution of $1.7 \mathrm{meV}$ are indicated by crossed symbols. The inset shows the bulk structure factor of liquid In as well as a sketch of the sound dispersion up to the quasi-Brillouin zone edge (dashed line). higher than the isothermal one. This is a common phenomenology, observed in many other liquids in the elastic regime, where the relaxations occur on time scales larger than the inverse of the characteristic frequency $1 / \Omega_{L}(Q)$. Furthermore, the longitudinal sound dispersion in surface liquid indium becomes slightly lower than in the bulk with increasing $Q$. Finally, the isothermal and infinite-frequency dispersions are systematically lower at the surface.

The form of the sound dispersion curves can be understood in analogy to crystalline systems. If the first sharp diffraction peak in the liquid structure factor is interpreted similar to a Bragg reflection in a crystalline system, the sound dispersion should exhibit a simple sinusoidal shape up to the Brillouin zone edge at half the distance in reciprocal space (see the sketch in the inset of Fig. 3). The data points deduced from the IXS spectra follow nicely this simple and model-independent picture up to $Q=11.5 \mathrm{~nm}^{-1}$ (dashed line) at half the distance to the structure factor maximum of liquid indium at $Q_{\max }=$ $23 \mathrm{~nm}^{-1}$.

The structural relaxation time, $\tau_{\alpha}$, despite the quite large error bars, reveals differences as well (see Fig. 4). From a weighted average of $\tau_{\alpha}$ one obtains $0.37 \pm 0.05$ ps for the bulk and $0.9 \pm 0.2 \mathrm{ps}$ for the surface sample. Conversely, less significant differences have been found for $\tau_{\mu}$ (see Fig. 4).

A further quantity that can be derived from the fitting parameters is the $Q$-dependent generalization of the longitudinal viscosity, $\eta_{L}(Q)$ :

$$
\eta_{L}(Q)=\frac{\rho}{Q^{2}}\left[\Delta_{\alpha}^{2}(Q) \tau_{\alpha}(Q)+\Delta_{\mu}^{2}(Q) \tau_{\mu}(Q)\right],
$$

where $\rho$ is the indium mass density. As for $\tau_{\alpha}$, the values of $\eta_{L}(Q)$ are systematically higher for surface liquid indium [see Fig. 5(a)].

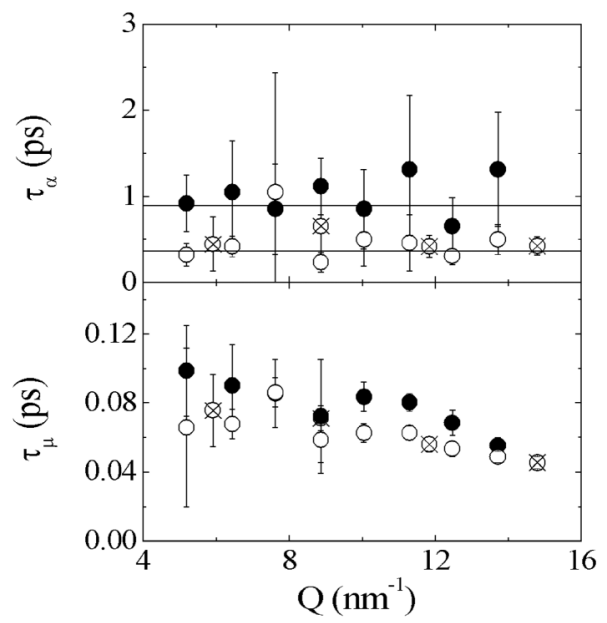

FIG. 4. Structural (top panel) and microscopic relaxation time (bottom panel) of liquid indium in bulk (open symbols) and surface-sensitive geometry (closed symbols) as a function of momentum transfer $Q$. The bulk results obtained with a higher energy resolution of $1.7 \mathrm{meV}$ are indicated by crossed symbols. The horizontal lines in the top panel are the weighted average. 


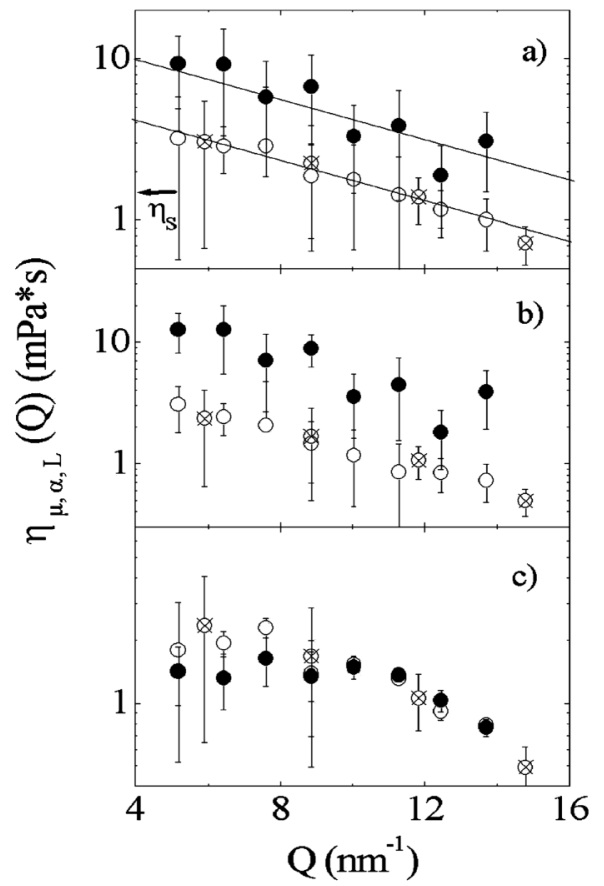

FIG. 5. (a) Longitudinal viscosity of liquid indium (top panel). The lines are interpolations of the data while the arrow indicates the $Q=0$ value of the shear viscosity [17]. Structural (b) and microscopic (c) components of the longitudinal viscosity of liquid indium in bulk (open symbols) and surface-sensitive geometry (closed symbols) as a function of momentum transfer $Q$. Bulk results obtained with a higher energy resolution of $1.7 \mathrm{meV}$ are indicated by crossed symbols.

The $Q$ dependence of $\eta_{L}(Q)$, can be described by an exponential decay: $\eta_{L}(Q)=\eta_{L} e^{-A Q}$. Fitting the experimental data with this function we can derive the value of the longitudinal viscosity at $Q=0$ : i.e. $\eta_{L}$. The obtained results are $7.4 \pm 0.2 \mathrm{mPas}$ for the bulk and $22 \pm 3 \mathrm{mPas}$ in the subsurface regime with $\Lambda=4.6 \mathrm{~nm}$ (compared to $2.2 \pm 3 \mathrm{mPa}$ s for the bulk shear viscosity). Figures 5(b) and 5(c) summarize the contributions of the structural and instantaneous relaxation, respectively. It is evident that the microscopic contribution is identical in the two cases, while the structural contribution is much larger at the surface. Since $\Delta_{\alpha}^{2}(Q)$ changes by only $\sim 10 \%$ (reflecting the softening of the limiting sound dispersions) from the bulk to the surface-sensitive measurement, such a significant increase of the longitudinal viscosity at the surface must be attributed to the slowing down of the collective dynamics associated to the structural relaxation process.

Finally, we would like to address the possible contribution of capillary waves to the IXS spectra. A quantitative calculation based on the formalism by Mora and Daillant [16] showed that the capillary waves are strongly overdamped in the probed $Q$ range with a negligible contribution to the scattering cross section of $3 \%$.
In conclusion, we have performed a study of the highfrequency dynamics in the subsurface and the bulk region of a metallic liquid. The analysis of the inelastic x-ray scattering spectra, based on a generalized hydrodynamic theory, points to a decrease of $\sim 10 \%$ of the sound dispersions, which is particularly visible at high $Q$, and an increase of the longitudinal viscosity by a factor of 3 in the subsurface region. The generalized hydrodynamic theory, where viscous dissipations are related to the relaxation processes, allows us to propose the mechanism responsible for the observed increase in the longitudinal viscosity. This high viscosity in the subsurface region is attributed to the slowing down of the collective dynamics associated with the structural relaxation process, since no differences have been observed in the microscopic relaxation. In turn, this can be related to the phenomenon of layer stratification, observed in the near-surface region [2], which is characterized by a density gradient normal to the surface, and a density change inside these layers compared to the bulk value. The increase in the longitudinal viscosity and the slowing down of the longitudinal dynamics casts a strong boundary condition on the detailed understanding of the density anisotropy near liquid surfaces. Our results should therefore stimulate further theoretical modeling of these processes.

Finally, this work clearly demonstrates the feasibility of IXS experiments on liquids in surface-sensitive geometry, opening up the possibility to investigate the collective dynamics of liquids in the subsurface region at interatomic length and time scales.

[1] O. M. Magnussen et al., Phys. Rev. Lett. 74, 4444 (1995).

[2] H. Tostmann et al., Phys. Rev. B 59, 783 (1999).

[3] O. G. Shpyrko et al., Science 313, 77 (2006).

[4] J. Penfold, Rep. Prog. Phys. 64, 777 (2001).

[5] G. Ruocco and F. Sette, J. Phys. Condens. Matter 11, R259 (1999).

[6] T. Scopigno, G. Ruocco, and F. Sette, Rev. Mod. Phys. 77, 881 (2005).

[7] U. Balucani and M. Zoppi, Dynamics of the Liquid State (Clarendon, Oxford, 1983).

[8] J.P. Boon and S. Yip, Molecular Hydrodynamics (McGraw-Hill, New York, 1980).

[9] T. Scopigno, U. Balucani, G. Ruocco, and F. Sette, Phys. Rev. Lett. 85, 4076 (2000).

[10] T. Scopigno, U. Balucani, G. Ruocco, and F. Sette, Phys. Rev. E 63, 011210 (2000).

[11] T. Scopigno, U. Balucani, G. Ruocco, and F. Sette, Phys. Rev. E 65, 031205 (2002).

[12] A. Monaco et al., J. Chem. Phys. 120, 8089 (2004).

[13] G. Ruocco et al., Phys. Rev. Lett. 84, 5788 (2000).

[14] O. J. Kleppa, J. Chem. Phys. 18, 1331 (1950).

[15] M. J. Duggin, J. Phys. F 2, 433 (1972).

[16] S. Mora and J. Daillant, Eur. Phys. J. B 27, 417 (2002).

[17] S. J. Cheng et al., Mater. Lett. 57, 4191 (2003). 